\title{
Discrete Element Simulation of Vibration Density Screen
}

\section{ZHOU Ziqiang ${ }^{1, a}$, DAI Guohong ${ }^{2, b}$, GUO Jian ${ }^{3, c}$, ZHANG Xiangyan ${ }^{3, d}$}

${ }^{1}$ School of Mechanical Engineering, Changshu Institute of Technology, Changshu 215500, China;

${ }^{2}$ Postdoctoral Workstation of Jiangsu Huahong Technology Stock Co. Ltd. , Wuxi, China

${ }^{3}$ Jiangsu Key Laboratory of Recycling and Reuse Technology for Mechanical and Electronic Produ cts, Changshu 215500, China

Azzq_rrme@163.com, bdgh@cslg.cn, 'guojian4307@163.com, ${ }^{\mathrm{d}}$ zhangxiangyancumt@163.com

Keywords: EDEM; Numerical simulation; Non-metallic shredder residue; Sorting.

Abstract. For the aim of sorting non-metallic shredder residue of body of end of life vehicle after disassembly, an innovative density screen is proposed. In this paper, the model of throwing and sliding of plastic and glass scrap of material for this equipment was defined. And then, the numerical simulation of vibration sorting process was carried out by the software of EDEM. The result of simulation showed that, plastic particle and glass particle can be separated at certain frequency and amplitude by this method.

\section{Introduction}

Currently, in the disassembly and recycling process of end-of-life vehicles, the vehicle body is generally subjected to packaging, crushing and separation process after disassembly operations ${ }^{[1]}$. Magnetic separation and eddy current technologies are adopted in the separation process to separate non-ferrous metal materials, such as steel, aluminum and copper. However, non-metallic materials, such as rubber, plastics, are treated as wastes. Conversely, using mechanical vibration technology to separate these non-metallic materials is a kind of method with low secondary pollution and high separation efficiency. However, the technical parameters of vibration separation equipment have major impact on separation efficiency. Therefore, using computer simulation methods for the optimal parameter design of separation screens is essential. We use discrete element simulation software EDEM to perform numerical simulation and analysis for separation screens under different frequencies, vibration amplitudes and vibration direction angles. Then, the process parameters of density separation screens are obtained.

\section{Model of material on the screen surface}

After materials enter the separation device, they perform sliding and throwing movements on the screen surface, thus making them move forward or backward on the screen plate. The movement model is shown in Fig. 1, where $\mathrm{F}$ is the vibration direction of screen plate; $\mathrm{X}$ direction is the tangential direction of screen plate; $\mathrm{Y}$ direction is the normal direction; and $\mathrm{G}$ is the gravity of materials ${ }^{[4]}$.

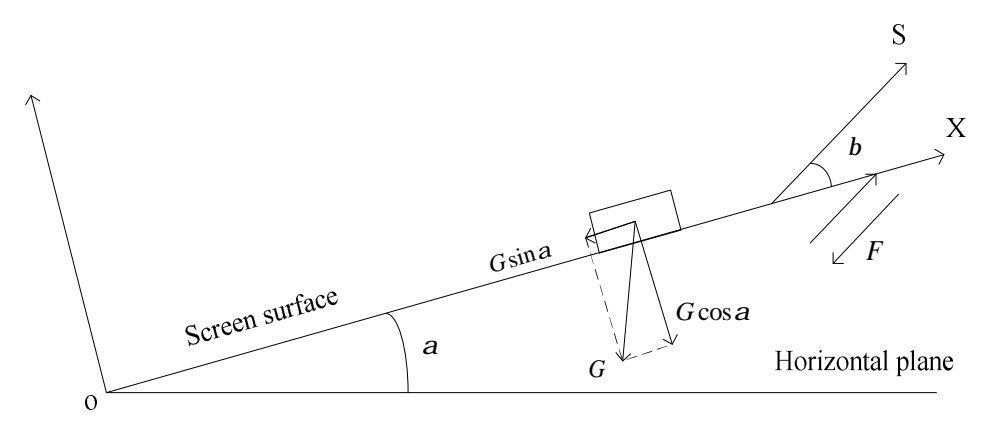

Note: $F$-Exciting force; $\beta$-Vibration direction angle; $\alpha$-Screen surface inclination; $G$-Gravity

Fig.1 Vibration Density Screen device diagram 
The working surface of the vibration density screen conducts simple harmonic movement along the vibration direction. According to kinetic analysis, the displacement equation of the working surface of the density screen is

$$
\begin{aligned}
S & =A \cdot \operatorname{Sin} \omega t \\
v & =\omega A \cdot \operatorname{Cos}(\omega t) \\
a & =-\omega^{2} A \cdot \operatorname{Sin}(\omega t) \\
K & =\frac{\omega^{2} A}{g} \\
D & =\frac{\omega^{2} A}{g} \cdot \frac{\operatorname{Sin} \beta}{\operatorname{Cos} \alpha}
\end{aligned}
$$

where

$K$ denote the vibration amplitude;

$D$ denote the throwing index; when it is larger than 1, materials can be thrown up;

$A$ denote the vibration amplitude of the separation screen;

$\omega$ denote the angular velocity of the vibration motor;

$\alpha$ denote the inclination of the screen surface;

$\beta$ denote the vibration direction angle, $\left({ }^{\circ}\right)$;

g denote the acceleration of gravity, $\left(g=9.81 \mathrm{~m} / \mathrm{s}^{2}\right)$.

A three-dimensional model of the vibration density separation screen is demonstrated in Fig. 2. The inclination angles of the three screen plates are all $9^{\circ}$. The lengths of the upper, middle, and lower screen plates are $300 \mathrm{~mm}, 250 \mathrm{~mm}$, and $250 \mathrm{~mm}$, respectively. To increase the computation speed, the widths of the three screen plates are set to $200 \mathrm{~mm}$, with the intervals set to $70 \mathrm{~mm}$.

The sorting process is realized through the contact and collision between particles and screen plates, so that some particles move upward or downward and different particles are separated. To correctly describe the inter-particle collision and the collision between particles and screen plates, the particle discrete element method is used: elastic - damping - friction - sliding contact mechanical model ${ }^{[2,5]}$, as shown in Fig. 3.

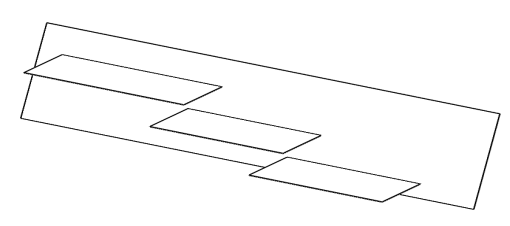

Fig.2 3D Model of Vibration Density Screen

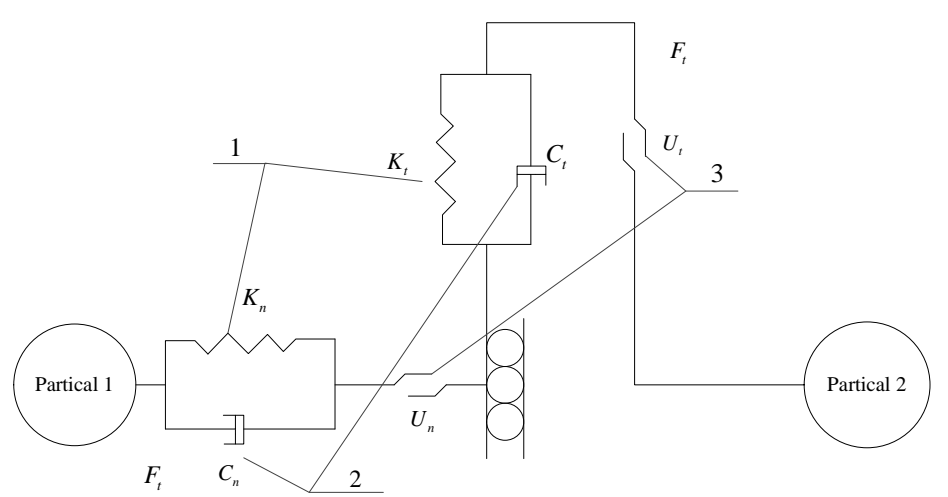

Note: $K_{n}$-Normal stiffness; $K_{t}$-Tangential stiffness; $C_{n}$ - Normal damping; $C_{t}$ - Tangential damping; $\mu$ - Friction coefficient; $F_{n}$-Normal force; $F_{t}$-Tangential force; 1. Particle stiffness (spring); 2. Damper; 3. Slider; 4. Friction device

Fig. 3 Contact mechanics model 
According to the calculation of the contact force, the following is obtained:

$$
\begin{aligned}
& F_{n i j}=\left(-k_{n} \alpha^{\frac{3}{2}}-\eta_{n i} G n\right) n \\
& F_{t i j}=-k_{t} \delta-\eta_{t j} G_{c t}
\end{aligned}
$$

Where

$\alpha$ denote the overlap amount of particles in normal direction;

$G$ denote the relative velocity between particle $i$ and particle $j ; G=v_{i}-v_{j}$;

$\mathrm{n}$ denote the unit vector from the Spherical center of particle $i$ to the Spherical center of particle $j$;

$\eta_{n i}$ and $k_{n}$ denote the normal damping coefficient and normal elastic coefficient of particle $i$, respectively;

$\eta_{t j}$ and $k_{t}$ denote tangential damping coefficient and tangential elastic coefficient, respectively;

$G_{c t}$ denote the slip velocity of the contact point between two balls;

$\delta$ denote the tangential displacement of the contact point between two balls.

$\mathrm{k}_{n}, \mathrm{k}_{t}$ and $G_{c t}$ denote given by the following equations:

$$
\begin{aligned}
& k_{n}=\frac{4}{3}\left(\frac{1-v_{i}^{2}}{E_{i}}+\frac{1-v_{j}^{2}}{E_{j}}\right)^{-1}\left(\frac{a_{i}+a_{j}}{a_{i} a_{j}}\right)^{\frac{-1}{2}} \\
& k_{t}=8 a^{\frac{1}{2}}\left(\frac{1-v_{i}^{2}}{G_{i}}+\frac{1-v_{j}^{2}}{G_{j}}\right)^{-1}\left(\frac{a_{i}+a_{j}}{a_{i} a_{j}}\right)^{\frac{-1}{2}} \\
& G_{c t}=G-(G n) n+a_{i} \Omega_{i} \times n+a_{j} \Omega_{j} \times n
\end{aligned}
$$

where

$V$ and $E$ denote the Poisson's ratio and elastic modulus of the particulate material, respectively; $a$ denote the radius of particles;

The subscriptions $i$ and $j$ represent the contacting particles $i$ and $j$, respectively;

$G_{i}$ and $G_{j}$ denote the shear modulus of particles $i$ and $j$, respectively;

$a_{i}$ and $a_{j}$ denote the radius of particles $i$ and $j$, respectively;

$\Omega_{i}$ and $\Omega_{j}$ denote the angle velocities of particles $i$ and $j$, respectively.

\section{Simulation with EDEM}

To increase the calculation speed of simulation, the glass particles and plastic particles in Non-metallic of ELV (End of life vehicle) residue are considered as the objects to be screened, without considering other impurities. Glass and plastic both have irregular flat-shaped profiles, as shown in Fig. 4. The physical parameters of glass, plastic and screen surface (steel) are shown in Table 1. The coefficients of restitution of all materials are calibrated as: 0.5 between glass and glass; 0.6 between glass and plastic; 0.3 between glass and screen surface; 0.45 between plastic and screen surface. The coefficients of static friction of all materials are calibrated as: 0.154 between glass and glass; 0.2 between glass and plastic; 0.154 between glass and screen surface; 0.5 between plastic and screen surface. The rolling friction coefficients of all materials are calibrated as: 0.1 between glass and glass; 0.01 between glass and plastic; 0.1 between glass and screen surface; 0.01 between plastic and screen surface. The drop heights of glass and plastic are $40 \mathrm{~mm}$, with a speed of $0.05 \mathrm{~m} / \mathrm{s}$. The producing speeds of glass and plastic are both 25 pieces per second. The generation time of particles is $5 \mathrm{~s}$, and the simulation time is $13.5 \mathrm{~s}$ in total. 

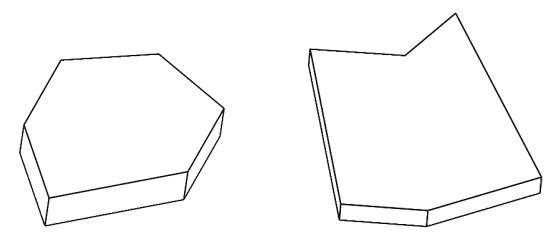

Fig.4 3D Model of shredded materials

Table.1 Material physics properties

\begin{tabular}{llll}
\hline Particle & Poisson's ratio & Shear modulus $/ \mathrm{Mpa}$ & Density $/\left(\mathrm{kg} \cdot \mathrm{m}^{-3}\right)$ \\
\hline Glass & 0.25 & 220 & 2465 \\
\hline Plastic & 0.3897 & 862.2 & 1400 \\
\hline
\end{tabular}

The calculation equation of the time steps is obtained according to Rayleigh wave method

$$
T=\frac{\pi R}{0.163 v+0.877} \sqrt{\frac{\rho}{G}}
$$

where

$R$ denote the particle radius $(m)$;

$\rho$ denote the particle density $\left(\mathrm{kg} \cdot \mathrm{m}^{-3}\right)$;

$G$ denote shear modulus (Mpa);

$V$ is the Poisson's ratio.

In the simulation of EDEM, the numbers of glass and plastic particles on the screen surface, the trajectories, and the number of particles falling into the front and back troughs can be displayed in real time. After simulation, statistical analysis is performed for the numbers of glass and plastic particles falling into the front and back troughs ${ }^{[6]}$.

Motion simulation of particles on screen surface.By referring to relevant documents and manuals, it can be concluded that the vibration amplitude, frequency and vibration direction angle have the most significant impact on screen separation. Therefore, these three parameters are the parameters to be optimized in analyzing the screen separation process.

Effect of vibration amplitude on separation. Under the condition that the frequency is $12 \mathrm{~Hz}$, and the vibration direction angle is , the vibration amplitudes of 4, 5, and $6 \mathrm{~mm}$ are selected for simulation. Fig. 5 shows the $\mathrm{X}$ components of speed for plastic particles from 0s to the end of simulation (13.5s). The speed variation from the moment when materials drop on the screen surface to $3.17 \mathrm{~s}$ is the same as that from $3.17 \mathrm{~s}$ to the moment when all the plastic particles drop on the screen surface $(5.55 \mathrm{~s})$.

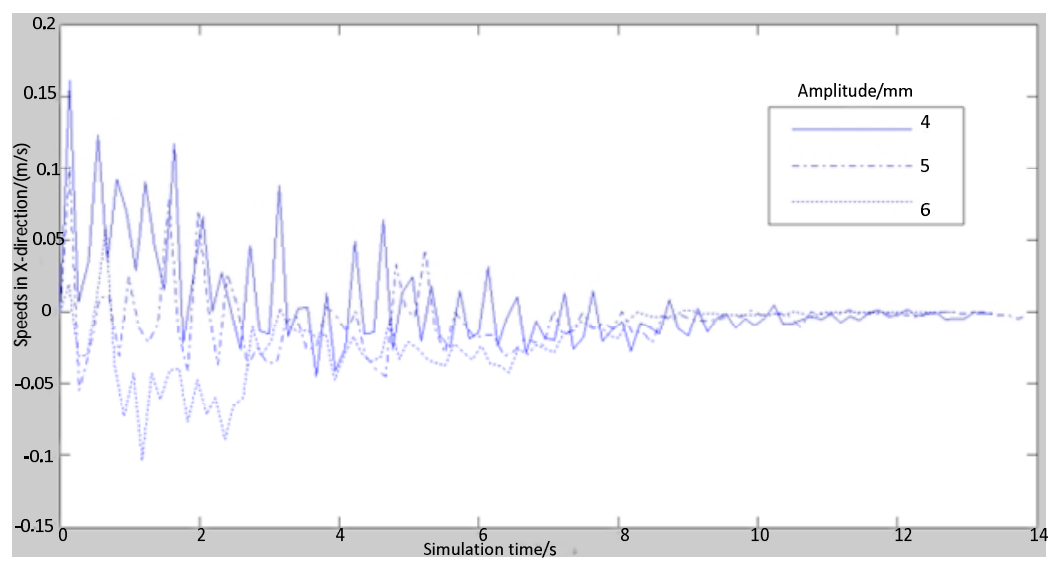

Fig. 5 Speed variation in $\mathrm{X}$ direction of plastic particles under different amplitudes

During the period from the phase when the plastic particles start to contact the screen surface to the phase when all particles fall into the front trough, the average speeds in X-direction for amplitudes of 4,5 , and $6 \mathrm{~mm}$ are $0.0063,-0.0056$, and $-0.0189 \mathrm{~m} / \mathrm{s}$, respectively. After all plastic particles fall, the 
particle layer becomes thin and the speed increases with the proceeding of screening. When most plastic particles fall into the front trough, the speed of the rest plastic particles decreases dramatically. When the amplitude is $6 \mathrm{~mm}$, all the plastic particles fall into the front trough at $9.42 \mathrm{~s}$, with 3 glass particles falling into the front trough. When the amplitude is $5 \mathrm{~mm}$, all the glass particles fall into the back trough at $8.81 \mathrm{~s}$, with 6 plastic particles still on the screen surface. When the amplitude is $4 \mathrm{~mm}$, all the glass particles fall into the back trough at 8.09s, with 20 plastic particles still on the screen surface. It can be concluded that the larger the amplitude, the higher the forward speed, and the shorter the time for separating plastic particles. However, when the amplitude is $6 \mathrm{~mm}$, there are 3 glass particles falling into the front trough, causing impurity in plastic particles, while when the amplitude is 4 or $5 \mathrm{~mm}$, there is no loss.

\begin{tabular}{llll}
\multicolumn{4}{c}{ Table 2 Separation rate of materials with different amplitudes } \\
\hline & $4 \mathrm{~mm}$ & $5 \mathrm{~mm}$ & $6 \mathrm{~mm}$ \\
\hline Plastic & $92.8 \%$ & $91.2 \%$ & $92.8 \%$ \\
\hline Glass & $100 \%$ & $100 \%$ & $97.6 \%$ \\
\hline
\end{tabular}

It can be concluded from Table 2 that the larger the amplitude is, the lower the separating ratio of glass particles is. The separating ratio of plastic particles for $4 \mathrm{~mm}$ is the same as that of $6 \mathrm{~mm}$. However, the separating ratio of glass particles for $4 \mathrm{~mm}$ is relatively higher. Therefore, in the cases where the other conditions are the same, the amplitude of $4 \mathrm{~mm}$ is conducive for separating the two kinds of particles.

Effect of frequency on separation. It can be concluded in the analysis of Section 3.2.1, taking into account both separation efficiency and separation impurities, the performance of amplitude $4 \mathrm{~mm}$ is relatively satisfactory. Therefore, the amplitude of $4 \mathrm{~mm}$ is selected when analyzing the effect of frequency on separation performance, with vibration direction angle set to . The simulation is carried out for frequencies of $12,16.167$, and $23.833 \mathrm{~Hz}$.

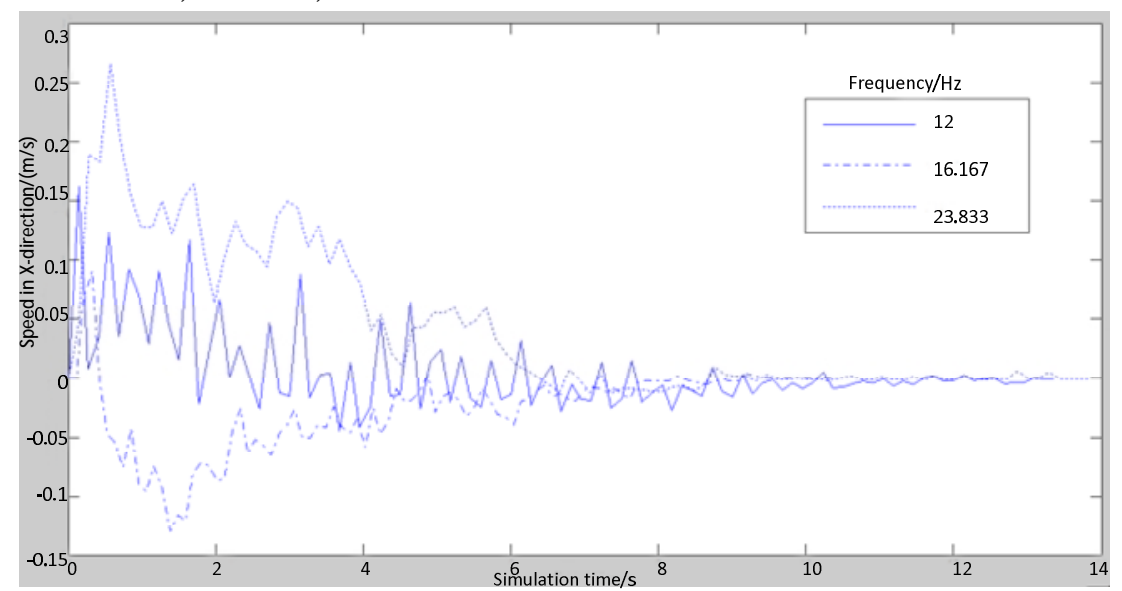

Fig.6 Speed variation in $\mathrm{X}$ direction of plastic particles under different frequencies.

It is clear in Fig. 6 that in case where the other conditions are the same, with the increase of frequency, the forward movement speed of particles increases. The X-direction speeds are 0.0063, -0.0268 , and $0.0419 \mathrm{~m} / \mathrm{s}$ for frequencies $12,16.167$, and $23.833 \mathrm{~Hz}$, respectively. When the frequency is $23.833 \mathrm{~Hz}$, there are 8 plastic particles on the screen surface without falling into the front trough at the end of simulation (at 13.5s). At this moment, the separating ratio of plastic particles is the lowest. However, all the glass particles have fallen into the back trough. When the frequency is $16.167 \mathrm{~Hz}$, all the plastic and glass particles have fallen into the front and back troughs by 10.34s. However, the separating ratio of glass particles is the lowest, while that of plastic particles is the highest. When the frequency is $12 \mathrm{~Hz}$, all the glass particles have fallen into the back trough by $8.09 \mathrm{~s}$, while most plastic particles have fallen into the front trough by $13.25 \mathrm{~s}$. The combined separating ratio of the plastic and glass particles is the highest. 
Effect of vibration direction angle on separation. It can be concluded from the two previous groups of simulation experiments that under the condition that the amplitude is $4 \mathrm{~mm}$ and the frequency is $2 \mathrm{~Hz}$, the vibration direction angles of $40^{\circ}, 45^{\circ}, 50^{\circ}, 55^{\circ}$ and $60^{\circ}$ are selected.

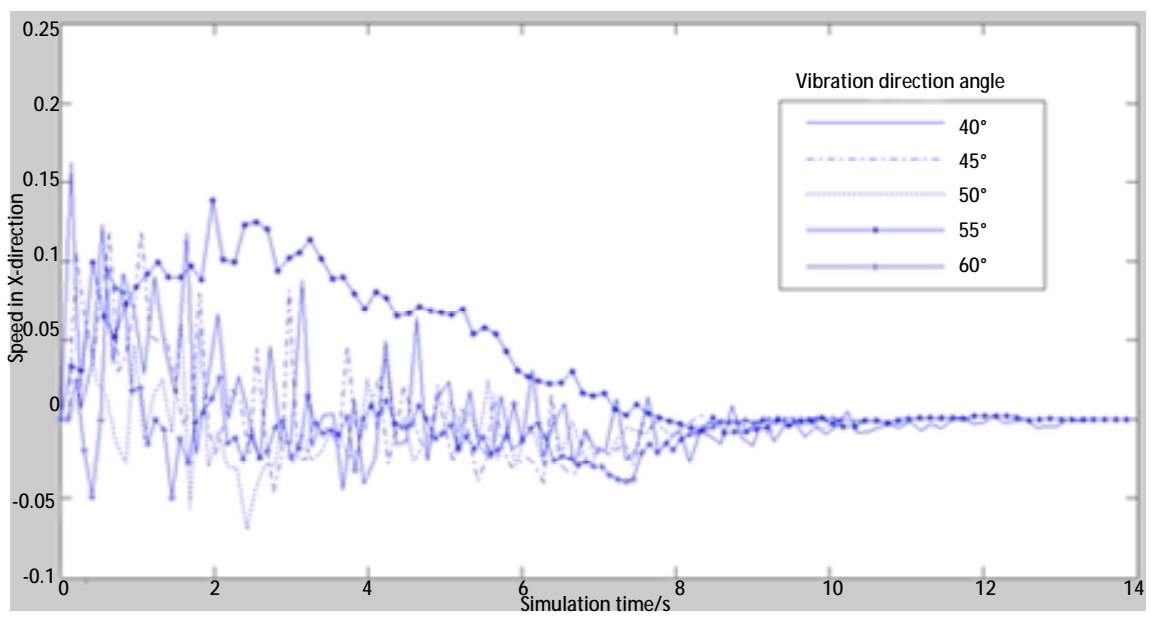

Fig.7 Speed variation in $\mathrm{X}$ direction of plastic particles under different vibration direction angles

It is clear in Fig. 7 that, the separating ratio is the lowest when . There are 3 plastic particles and 4 glass particles on screen surface at 13.5s. When the inclination is, the separating ratio is the highest. However, there is one glass particle falling into the front trough, causing loss. In the range of $40^{\circ}$ to $60^{\circ}$, with the increase of vibration direction angle, the separating ratio of glass particles decreases. The separating ratio of plastic particles reaches maximum at $50^{\circ}$, while minimum at $60^{\circ}$

\section{Conclusions}

In the cases where the other conditions are the same, with the increase of frequency and amplitude, (i.e., the throwing index and vibration strength increase), the particles' forward speed along the screen surface increases, and the separation efficiency improves. However, the increase of frequency and amplitude also causes the increase of impurities. Therefore, the increase of impurity should also be considered when the separation speed is increased.

In the cases where other conditions are the same, the forward speed of plastic particles reaches the maximum at the direction angle of $55^{\circ}$. For other angles, the fluctuation of the forward speed of plastic particles is large and within a certain range. When the vibration direction angle is the smallest, the throwing index is the lowest. The movement of plastic particles on the screen surface is mainly sliding, and the moving speed of the screen surface is just the moving speed of plastic particles. Therefore, when the angle is $40^{\circ}$, the speed fluctuation is the largest. With the increase of vibration direction angle, the throwing index increases, and the separation efficiency decreases. The separation efficiency reaches maximum when the vibration direction angle is $50^{\circ}$, with impurity content almost zero. Thus, it can be concluded by analyzing the simulation result that the best separation performance is obtained when the vibration amplitude is $4 \mathrm{~mm}$, the frequency is $12 \mathrm{~Hz}$, and the direction angle is $50^{\circ}$.

\section{Acknowledgments}

This work was financially supported by Science \& Technology Pillar Program of Jiangsu Province (No. BE2013060). Postdoctoral Research Program of Jiangsu Provinec (No.1401071C). 


\section{References}

[1] Muhamad Zameri, Mat Saman, Gordon N. Blount. End of life vehicles recovery: process description, its impact and direction of research[J]. Journal Mekanikal, 2006.1:40-52

[2] Sun Qi-cheng, Wang Guang-qian. An introduction to granular material mechanics[M].Beijing: Science Press, 2009:59-60.

[3] Li Hong-chang, Li Yao-ming, et al. Numerical simulation and analysis of vibration screening based on EDEM[J]. Transactions of the CSAE,2011,27(5):117-121.

[4] Wen Bang-chun, Liu Shu-ying. Modern vibrating screen technology and equipment design[M].Beijing: Metallurgical Industry Press,2013:94-110.

[5] Li-J, Webb-C, Pandiella-S S, et al. Discrete particle motion on sieves - a numerical study using the DEM simulation[J]. Powder Technology,2003,133:190-202.

[6] Hu Guo-ming, et al. Analysis and Simulation of Granular System by Discrete Element Method Using EDEM[M]. Wu han: Wuhan University of Technology Press,2010:261-264.) 